\title{
Quantum effects in the Aubry transition
}

\author{
Pietro Maria Bonetti $\odot,{ }^{1}$ Andrea Rucci $\odot,{ }^{2}$ Maria Luisa Chiofalo $\odot,{ }^{2, *}$ and Vladan Vuletić $\circledast^{3, \dagger}$ \\ ${ }^{1}$ Max Planck Institute for Solid State Research, Heisenbergstrasse 1, D-70569 Stuttgart, Germany \\ ${ }^{2}$ Dipartimento di Fisica “Enrico Fermi," Università di Pisa and INFN, Largo B. Pontecorvo 3, I-56127 Pisa, Italy \\ ${ }^{3}$ Department of Physics, MIT-Harvard Center for Ultracold Atoms and Research Laboratory of Electronics, Massachusetts Institute of \\ Technology, Cambridge, Massachusetts 02139, USA
}

(Received 28 August 2020; accepted 14 December 2020; published 12 January 2021)

\begin{abstract}
The Aubry transition between sliding and pinned phases, driven by the competition between two incommensurate length scales, represents a paradigm that is applicable to a large variety of microscopically distinct systems. Despite previous theoretical studies, it remains an open question to what extent quantum effects modify the transition, or are experimentally observable. An experimental platform that can potentially reach the quantum regime has recently become available at MIT in the form of trapped laser-cooled ions subject to a periodic optical potential [A. Bylinskii, D. Gangloff, I. Counts, and V. Vuletić, Observation of Aubry-type transition in finite atom chains via friction, Nat. Mater. 15, 717 (2016)]. Using path-integral Monte Carlo (PIMC) simulation methods, we analyze the impact of quantum tunneling on the sliding-to-pinned transition in this system and determine the phase diagram in terms of incommensuration and potential strength. We propose new signatures of the quantum Aubry transition that are robust against thermal and finite-size effects and that can be observed in future experiments.
\end{abstract}

DOI: 10.1103/PhysRevResearch.3.013031

\section{INTRODUCTION}

An intriguing feature of quantum many-particle systems is that they may undergo phase transitions even at zero temperature, driven by the competition between kinetic and interaction energies across a critical point [1]. While the transition can be influenced by a variety of parameters such as the dimensionality of the system, the strength and range of the interactions, or the amount of disorder, a particularly interesting situation arises when the phase transition is driven by the competition between two length scales. The latter mechanism gives rise to complex phenomena in a number of remarkably different physical environments, such as charge density waves [2], nanocontacts between solids [3], dislocations in crystals [4], adsorbed monolayers [5] in the form of noble gases on graphite substrates [6], biomolecular transport [7], emergence of chaotic structures in metal-insulator transitions in Peierls systems [8] and spin glasses [9], Josephson junctions [10], quantum pinning in strongly interacting bosonic fluids [11], and Meissner-to-vortex transition in bosonic ladders [12,13]. This nonexhaustive list illustrates how the concept of competing length scales underlies a wide range of phenomena from classical to quantum, with the latter prominently emerging in reduced dimensions, where quantum fluctuations and correlations are greatly enhanced.

\footnotetext{
*maria.luisa.chiofalo@unipi.it

†vuletic@mit.edu
}

Published by the American Physical Society under the terms of the Creative Commons Attribution 4.0 International license. Further distribution of this work must maintain attribution to the author(s) and the published article's title, journal citation, and DOI.
Various interesting behaviors can then emerge, depending on whether the two lengths are commensurate (C), i.e., in a rational ratio, or incommensurate (IC, irrational ratio), and how the incommensuration is accommodated by the system. Incommensurations can, for example, float on the commensurate phase [14], or instead occur as interstitial phases separating commensurate phases [6]. In general, a transition may occur from a pinned to a floating, translationally invariant phase. The incommensurate phase can be associated with a manifestation of translational invariance in the form a gapless excitation called phason, corresponding to superlubric sliding. In the commensurate phase, translational invariance is broken, and a gap appears in the excitation spectrum that corresponds to the energy required for the classical particle to climb the barrier. Above the transition point, no particles are found at the lattice maxima, as elucidated by Aubry [15]. Defects in the pinned phase can be in the form of walls, dislocations, or vortices [9].

The paradigmatic model to describe this general situation is a one-dimensional (1D) chain of particles connected by harmonic springs, separated at equilibrium by a distance $d$, and subjected to a substrate lattice with period $a$. This model has been independently proposed by Frenkel and Kontorova [16], and Frank and Van der Merwe [17] (FKVdM). In the FKVdM model, the competition is between the elastic potential, which favors a periodic structure with period $d$, and the lattice potential which tries to lock the particle positions at integer multiples of $a$. As a result, in the limit of small lattice potential $V$, the particles float on the lattice almost independently of the ratio $w \equiv d / a$ in the IC phase, while in the $\mathrm{C}$ phase, the particles localize near the lattice minima in a commensurate structure with an average spacing that is a rational multiple of $a$. The transition can be understood in more detail by introducing the average spacing $\bar{d}$ between 
the atoms in the chain and the winding number $\tilde{w}=\bar{d} / a$. At $V=0, \tilde{w}=w$, and the particles float. A finite lattice depth acts to localize the particles. nonrational values of $w$ are accommodated by an energy increase that maintains the winding number $\tilde{w}=w$, until there is sufficient energy to form a discommensuration. The C-IC transition predicted by Aubry [15] sets in as a second-order transition, and the winding $\tilde{w}$ jumps up to the next rational number. Below a critical lattice strength $V_{c}, \tilde{w}$ is irrational in finite intervals of $w$, forming a Cantor set with nonintegral fractal dimension [6], the interval size reducing to zero measure above threshold $V>V_{c}$. The critical lattice depth $V_{c}$ depends on the irrational value of the length ratio $d / a$, taking on its largest value at the golden ratio $(\sqrt{5}-1) / 2$. Notably, the transition survives even when the number of particles is finite [18].

The quantum version of the FKVdM model has also been investigated [19]. Quantum Monte Carlo (QMC) studies for up to 144 particles have revealed that quantum mechanics tends to smooth the onset of the Aubry transition [20]. Indicators of the transition have been defined in close analogy to the classical transition and include the hull function, the position variance of the particles, and the density-density correlation function. An effective Planck constant to measure the degree of quantum behavior has been identified [20,21]. Correlations in the $\mathrm{C}$ phase have been investigated via densitymatrix renormalization group (DMRG) [22] and path-integral molecular dynamics methods [23], while the IC phase has been studied by DMRG [24]. Excitations across the transition have been discussed in terms of a pinned instanton glass turning into a sliding phonon gas [21]. The effect of long-range interactions has been investigated in Ref. [25]. The formation of kinks, i.e., topological solitons, and kink-antikink pairs within a nonlocal FKVdM model in the presence of longrange, power-law interactions, has been characterized [26], also accounting for finite-size effects [27]. The relevance of the FKVdM-model to structural zigzag instabilities of ion strings [28] in optical resonators has also been pointed out [29-31]. Besides simulations, theoretical methods include the possibility of describing the C-IC transition in terms of an Ising model, as in the seminal work by P. Bak and R. Bruinsma [32].

Notwithstanding this wide range of previous studies, a number of crucial questions regarding the quantum Aubry transition remain open, such as the universality class of the transition, the relation between the $\mathrm{C}$-IC transition and the paradigm of many-body localization, the degree of universality for different types of interactions, and the general experimental feasibility of observing quantum effects in the FKVdM model in realistic systems of finite size and temperature.

In a recent experiment using a small chain of trapped $\mathrm{Yb}^{+}$ ions, Bylinskii et al. have observed an Aubry-like transition [33] at low temperatures where quantum effects may come into play by studying friction, and investigated the dependence on temperature [34] and commensurability [35]. In this finitesize system, the competition between the two different length scales, given by the period of the applied optical potential and the average interparticle spacing of the trapped particles, drives a transition between pinned and sliding arrangements. Using suitably devised atom-by-atom control and observation techniques, the transition from superlubricity to stick-slip has been investigated as a function of the height of the optical potential and the commensurability of the two length scales [30], thus demonstrating a versatile and controllable platform to explore the building blocks of friction at a nanoscopic level [36-39] over a wide temperature range. In a second iontrap experiment in two dimensions (2D) [40], the underlying nanofriction and transport processes have been investigated with atomic resolution using spectroscopic measurements, enabling the observation of a soft vibrational mode through the transition.

In this work, we investigate how to unequivocally identify quantum effects in the Aubry transition, and whether they can be observed in present finite-size ion-chain experiments that provide only an approximation to the FKVdM model. To this aim, we perform PIMC simulations reproducing the finite temperature and other conditions of the experiment [35], and propose new observables - never considered so far-as consistent indicators of emerging quantum behavior. We show that the quantum effects are sufficiently robust with respect to thermal fluctuations at the temperatures that have already been experimentally achieved, and that they can also be readily distinguished from finite-size effects already in the relatively small systems that are currently available.

The paper is organized as follows. In Sec. II, we present the system and the discuss the governing parameters. After introducing the methodological tools in Sec. III, we discuss in Sec. IV the simulation results and their theoretical implications, devoting Sec. V to a discussion how to observe quantum signatures in the actual experiment. We finally present conclusive remarks in Sec. VI.

\section{THE SYSTEM}

The system concept is outlined in Fig. 1. Laser-cooled, positively charged ions arranged in a 1D chain interact with each other via Coulomb repulsive forces while being overall

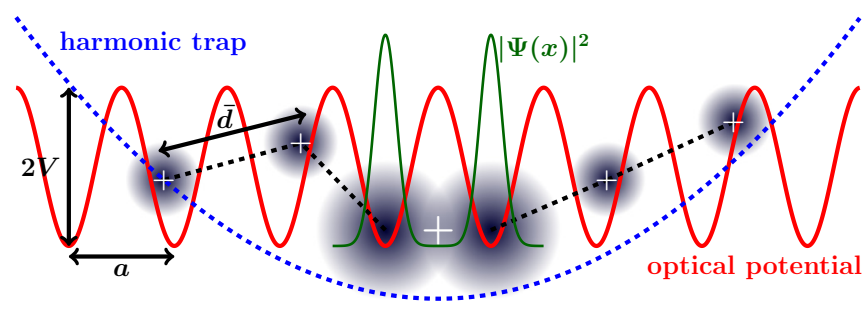

FIG. 1. Concept for experimental realization of the quantum Aubry transition with laser cooled trapped ions subject to an opticallattice potential following Ref. [33]. $N$ ions with charge $+|e|$ are separated on average by a distance $\bar{d}$ that results from the combined action of the mutual Coulomb repulsion and the external harmonic confinement. Overlaid is a periodic optical lattice with lattice constant $a$ and height $V$. The length scales $a$ and $\bar{d}$ are in an irrational or incommensurate ratio. The ions may float on the periodic lattice in the incommensurate phase for $V$ below a critical value $V_{c}$, or pin into commensurate pieces separated by incommensurations for $V>V_{c}$. Quantum effects are driven by tunneling between two neighboring lattice sites. Here, the quantum probability density $|\Psi(x)|^{2}$ for the central particle is sketched, as calculated with a PIMC simulation. 
confined in a harmonic potential characterized by a trap frequency $\omega_{0}$. The combined action of the Coulomb repulsion and the harmonic confinement sets the ion arrangement with an average interparticle distance $\bar{d}$ defined by

$$
\bar{d}=\sum_{i=-(N-1) / 2}^{(N-3) / 2} \frac{\left|x_{i+1}-x_{i}\right|}{N-1}
$$

where $x_{i}$ is the position of the $i$ th ion of the system with $N$ particles. An optical lattice provides the static periodic potential with height $V$ and period $a$, with the two length scales $\bar{d}$ and $a$ being in a (quasi-) incommensurate ratio. Notice that $\bar{d}$ is calculated in the absence of the lattice, i.e., for $V=0$. Depending on whether the lattice-potential height $V$ is below or above a critical value $V_{c}$, the ions either float on the lattice in a superlubric phase, or are pinned into the lattice wells, forming commensurate regions separated by incommensurate ones.

We thus consider the following Hamiltonian, in cgs units, precisely reproducing the experimental realization from Ref. [33]:

$$
\begin{aligned}
\mathcal{H}= & \sum_{i=-(N-1) / 2}^{(N-1) / 2}\left\{\frac{p_{i}^{2}}{2 m}+\frac{1}{2} m \omega_{0}^{2}\left(x_{i}-\frac{a}{2 \pi} \Phi\right)^{2}\right. \\
& \left.+V\left[1+\cos \left(\frac{2 \pi}{a} x_{i}\right)\right]\right\}+\sum_{i, j>i} \frac{e^{2}}{\left|x_{i}-x_{j}\right|} .
\end{aligned}
$$

The first term represents the kinetic energy of the $N$ ions having mass $m$, with $N$ being an odd number, the second term the harmonic confining potential with oscillator frequency $\omega_{0}$, while the third accounts for the periodic potential with height $V$ and period $a$, with a possible shift $\Phi$ between the minima of the harmonic trap and of the optical potential. The last term represents the Coulomb interactions between the $N$ ions, each possessing a charge $+|e|$. Whenever not explicitly specified, the value $\Phi=0$ is considered.

The system is thus characterized by two different length scales, namely $\bar{d}$ and $a$. Notice that, while the spacing $d_{i} \equiv$ $\left|x_{i+1}-x_{i}\right|$ between neighboring ions in the chain is nonuniform, the ratio $\bar{d} / a$ is sufficiently large that approximately the same incommensurability $\left(d_{i} / a \bmod 1\right)$ for all ions can be achieved by slight adjustment of the harmonic-trap potential [33]. The combined action of the Coulomb and harmonicoscillator potentials acts as a springlike internal force with effective spring constant $\gamma$ after linearization. As a result, $\bar{d}$ and $a$ define together the elastic energy

$$
E_{e}=\gamma\left(\frac{a}{2 \pi}\right)^{2} ; \quad \gamma=\frac{2 e^{2}}{\bar{d}^{3}},
$$

which will be used as our energy unit $E_{e}$ from now on. Notice that $E_{e}$ depends on the harmonic-oscillator frequency $\omega_{0}$ through the average ion spacing $\bar{d}$. In addition to the effective spring energy arising from the Coulomb potential, there are external forces due to the periodic potential, characterized by $K=V / E_{e}$ in dimensionless units, and due to the harmonic trap confinement.

To further account for the two length scales $\bar{d}$ and $a$, we introduce a third parameter $\Delta$, quantifying the incommensuration of the system via

$$
\Delta=1-\max _{\theta}\left\{\frac{1}{N} \sum_{j=-(N-1) / 2}^{(N-1) / 2} \cos \left(\frac{2 \pi}{a}\left\langle x_{j}\right\rangle+\theta\right)\right\},
$$

where $\left\langle x_{j}\right\rangle$ is the average position of the $j$ th particle measured at vanishing periodic potential $K=0$, and depends on the parameters of the harmonic potential. In this manner, $\Delta=$ 0 corresponds to a maximally commensurate system, while $\Delta=1$ corresponds to a maximally incommensurate situation. Overall, we see that the harmonic confinement plays three different roles: it stabilizes the internal repulsive forces, it produces a nonhomogeneity in the external forces, and it may be used to adjust the commensurability of the system.

We choose the same physical parameters to those typical in the experiment [35], so that $k_{B} T \simeq 0.01 \hbar \omega_{0}$, i.e., the lowest reachable temperature, and $a=185 \mathrm{~nm}$. Simulations at different temperatures are performed in order to check the robustness of quantum effects in the presence thermal fluctuations. The other parameters are varied in the following ranges: $\omega_{0} /(2 \pi)=2.09-2.11 \mathrm{MHz}$ and $V / k_{B}=0-1.60 \mathrm{mK}$. These ranges correspond to $\bar{d} / a \simeq 10^{3}$. Notice that despite this large ratio, the parameter that drives the system across the transition is the quantity $(\bar{d} / a \bmod 1)$, so that even small variations in $\bar{d}$ can significantly modify the system behavior.

\section{THE METHOD}

In order to determine the ground state of Hamiltonian (2), we resort to a path-integral Monte Carlo (PIMC) simulation at finite temperature $T$ [41], where the path integrals are computed within a Markov-chain Monte Carlo (MCMC) simulation [41]. Finite temperature enters after executing a Wick rotation $(t \rightarrow i \tau)$ in the classical action. Thus, time evolution occurs along an imaginary time axis with length $\hbar / k_{B} T$, and periodic boundary conditions. In this manner, each particle is characterized by a loop in imaginary time. The configurations, i.e., the collection of all particle paths, follow a probability distribution given by the Boltzmann weight. Configurations are then sampled according to the distribution, and the observables are calculated on those samples. In performing this operation, we take special care of autocorrelation effects of next-observable measurements and of time discretization (see Appendix A). In particular, all the presented data result from having performed simulations for three different values of the time-step $\tau$, extrapolating the limit $\tau \rightarrow 0$ from a linear fit. Finally, special care has also been taken in order to efficiently deal with tunneling processes arising when the minimum of the harmonic trap coincides with a maximum of the optical potential [i.e., when $\Phi=2 n \pi$ in Eq. (2)]. For more details on this technical choice, we refer to Appendix A.

\section{RESULTS}

\section{A. Emergence of quantum effects and bimodal probabilities}

In order to mark the onset of the transition, an order parameter needs to be identified. In particular, we seek a quantitative parameter that indicates whether the single particle wave function has a Gaussian or a bimodal shape. To this end, we 


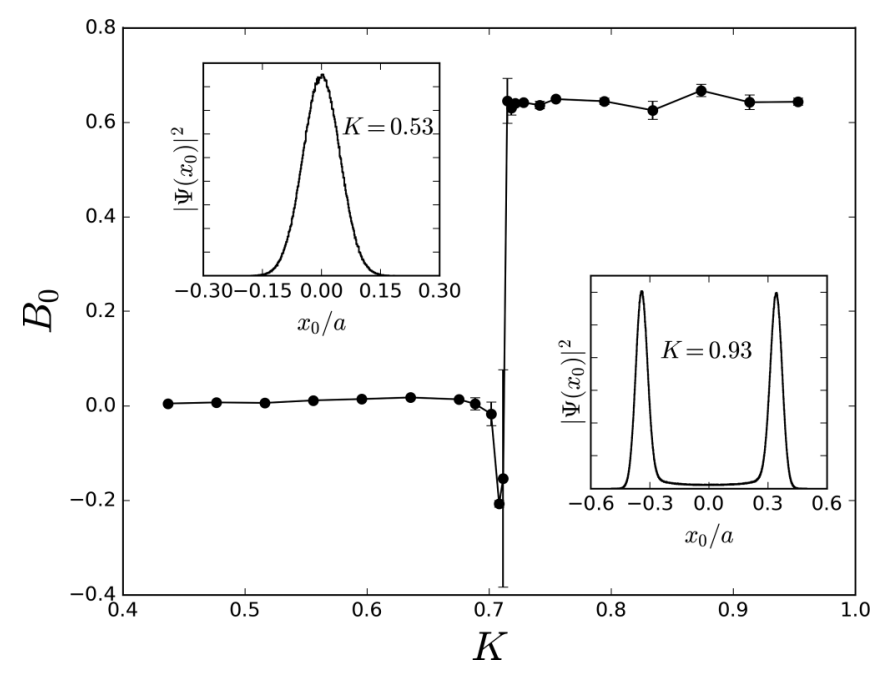

FIG. 2. Characterizing quantum effects via the Binder cumulant $B_{0}$ in a near-commensurate situation $(\Delta \approx 0.22)$. Behavior of $B_{0}$ [Eq. (5)] for the probability distribution of the central particle as a function of the scaled optical-lattice depth $K$ in a fully commensurate system. Notice the sharp increase of $B_{0}$ at the value $K \simeq K_{c} \simeq 0.7$. For low values of the potential, $B_{0} \simeq 0$, indicating a Gaussian-like distribution, while for large $K$ values we find $B_{0} \simeq 2 / 3$, characteristic of a bimodal distribution. Upper left inset: Gaussian-like probability distribution (modulus squared of the single particle wave function) for $K<K_{c}$. Lower right inset: bimodal distribution for $K>K_{c}$.

consider the Binder cumulant (BC), defined as

$$
B_{j} \equiv 1-\frac{\left\langle\left(x_{j}-\bar{x}_{j}\right)^{4}\right\rangle}{3\left\langle\left(x_{j}-\bar{x}_{j}\right)^{2}\right\rangle^{2}},
$$

where $\bar{x}_{j}=\left\langle x_{j}\right\rangle$ and the averages are to be calculated on the single-particle probability distribution $\varrho_{j}(x)$. In our case, this is the probability density of a given ion, derived from the many-particle wave function that is traced over the other particles, the total number being $N=5$ as in the experiment [35]. In fact, the $\mathrm{BC}$ translates a qualitative shape of the wave function into a quantitative indicator. A Gaussian probability distribution is characterized by $B=0$, while a bimodal distribution composed of two symmetric Dirac delta functions yields $B=2 / 3$. The splitting of the single-peaked wave function into a bimodal shape as $K$ is increased is due to the suppressed probability of finding the particle near a lattice peak, in conjunction with quantum tunneling between two neighboring lattice minima. The $\mathrm{BC}$ is a quantity that can distinguish between spatial probability distributions with one or two peaks, thereby providing a clear signature of the emergence of quantum effects driven by tunneling.

We first test the suitability of the BC as an order parameter in a nearly commensurate situation. To this aim, we calculate the BC while varying $K$, and first analyze the behavior of the central particle, as shown in Fig. 2. The corresponding value $B_{0}$ of the $\mathrm{BC}$ is observed to sharply increase at $K_{c} \simeq 0.7$ towards the limiting value of $2 / 3$. As long as the system is in the sliding phase for $K<K_{c}$, the wave function also keeps the single-mode structure depicted in the top-left inset. In the opposite $K>K_{c}$ regime of the pinned phase, the bimodal
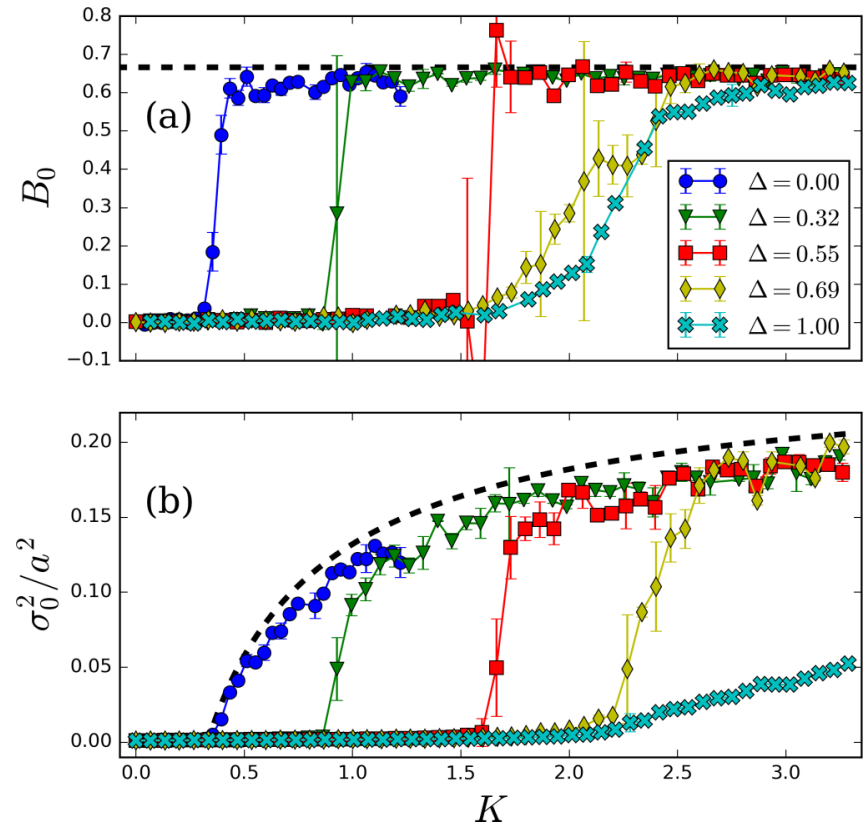

FIG. 3. Incommensuration effects. (a) Behavior of the Binder cumulant of the central particle, $B_{0}$, as a function of potential depth $K$ for different incommensuration parameter values $\Delta$ [Eq. (4)]. $\Delta=0$ corresponds to a maximally commensurate and $\Delta=1$ to a maximally incommensurate system. The potential configuration is the one in which the minimum of the trap coincides with an optical-lattice maximum. The dashed line represents the value $2 / 3$ expected for a bimodal distribution. (b) Variance $\sigma_{0}^{2}$ of the central particle under the same conditions as for (a). As for the Binder cumulant, we notice that larger incommensuration $\Delta$ moves the critical potential depth $K_{c}$ to larger values and softens the transition. The dashed line represents the variance for the classical position of the central particle as described in the text. It is interesting how for all incommensurations, with the exception of $\Delta=1$, the variance approaches this universal classical behavior.

structure in the bottom-right inset is observed, as a signature of quantum tunneling leading to the localization in two wells.

Zooming in on the transition point, we notice a region where $B<0$. We find that when approaching the transition point, the tails of the probability distribution begin to extend, so that the fourth moment exceeds the second moment by more than a factor of 3 . However, near the transition, the PIMC convergence slows down, and statistical errors are larger, preventing us from further exploring the details of the probability distribution.

\section{B. The effects of incommensuration}

Having established that the $\mathrm{BC}$ provides a quantitative signature of the sliding-to-pinned transition, we now turn to explore the effects of incommensuration. Figure 3 displays the behavior of the $\mathrm{BC}$ of the central particle, $B_{0}$, as a function of the lattice potential strength $K$ for different values of the incommensuration parameter $\Delta$. We notice that a larger $\Delta$ increases the critical pinning potential $K_{c}$, i.e., the more incommensurate the system, the more it resists pinning. At the same time, the transition becomes less sharp compared to the corresponding commensurate case in Fig. 2. In fact, in 
the commensurate system the springs have no effect (they are neither compressed nor stretched), and all the particles move in unison but independently, reducing the transition to that of a single-particle. On the other hand, under incommensurate conditions $(\Delta \neq 0)$, it may happen that some of the particles do not undergo the pinning transition because they are at the minima of the optical lattice, while others are near the maxima. This requires larger values $K$ of the potential to complete the transition. Thus the transition region is widened as each particle becomes pinned at a different value of $K_{c}$. The smoothing is seen to persist in a ring geometry without the external confinement (see Sec. IV G below and Appendix B), and in the maximally incommensurate case has been observed also in Ref. [20]. The same smoothing does not happen in the classical Aubry model, where the transition is sharp and simultaneous for all particles [15].

Similar conclusions can be drawn from the position variance $\sigma_{0}^{2}=\left\langle x_{0}^{2}\right\rangle-\left\langle x_{0}\right\rangle^{2}$ of the central particle for $\Phi=0$. This variance is displayed in Fig. 3(b) as a function of $K$ for different incommensuration values $\Delta$. Above a critical value $K_{c}$ that agrees with that inferred from the $\mathrm{BC} B_{0}$, the variance $\sigma_{0}^{2}$ increases. As already pointed out in Ref. [20], $\sigma_{0}^{2}$ is a useful observable, although it cannot be considered, strictly speaking, an order parameter. We can compare the simulations of $\mathrm{Hu}$ et $\mathrm{al}$., performed with up to 144 particles connected by springs [20], with our five-particle system interacting via Coulomb forces, and find that the steepness of the transition is similar for comparable parameter values. We may thus infer that, at least for small incommensurations, our steep signature of the transition is robust against the effects of finite system size. Second, as with the BC behavior, larger values of the incommensuration parameter $\Delta$ move the critical $K_{c}$ to larger values, and broaden the transition.

However, two differences are evident when comparing the BC $\left[B_{0}\right.$ in Fig. 3(a) $]$ and the variance $\sigma_{0}^{2}$ of the central particle [Fig. 3(b)]. First, in the maximally incommensurate case with $\Delta=1$, the variance never reaches the limiting value, while the BC does. Second, for $\sigma_{0}^{2}$ the steepest behavior occurs for intermediate incommensuration, in contrast to the behavior for $B_{0}$.

The origin of this effect can be understood by calculating the classical position distribution of a single particle as a function of $K$. To this aim, we consider the many-particle potential $V\left(x_{-(N-1) / 2}, \ldots, x_{(N-1) / 2}\right)$, composed of external confinement, optical lattice, and Coulomb interactions, and minimize it over the variable set $\left\{x_{-(N-1) / 2}, \ldots, x_{(N-1) / 2}\right\}$, obtaining the values $\bar{x}_{i}$ of the particle positions that minimize $V$. Finally, we retain only the central particle position $\bar{x}_{0}$. In principle, the positions of the minima depend on the harmonic trap frequency, but we find that in the asymptotic limit of large $K$ this is not the case. The classical position of the central particle, calculated by the illustrated method, is in fact represented by the dashed line in Fig. 3, which evidently fits the numerical data in the $K \rightarrow \infty$ limit, without any fitting parameter. Indeed, when $K$ is increased above the critical value $K_{c}$, the central particle wave function resembles more and more a double delta distribution peaked at the classical minima of the many-particle potential $\pm \bar{x}_{0}$, i.e., $\Psi(x) \simeq 1 / 2\left[\delta\left(x-\bar{x}_{0}\right)+\delta\left(x+\bar{x}_{0}\right)\right]$, whose variance is indeed $\bar{x}_{0}^{2}$. In contrast, in the maximally incommensurate case $(\Delta=1)$, the central particle variance is evidently far

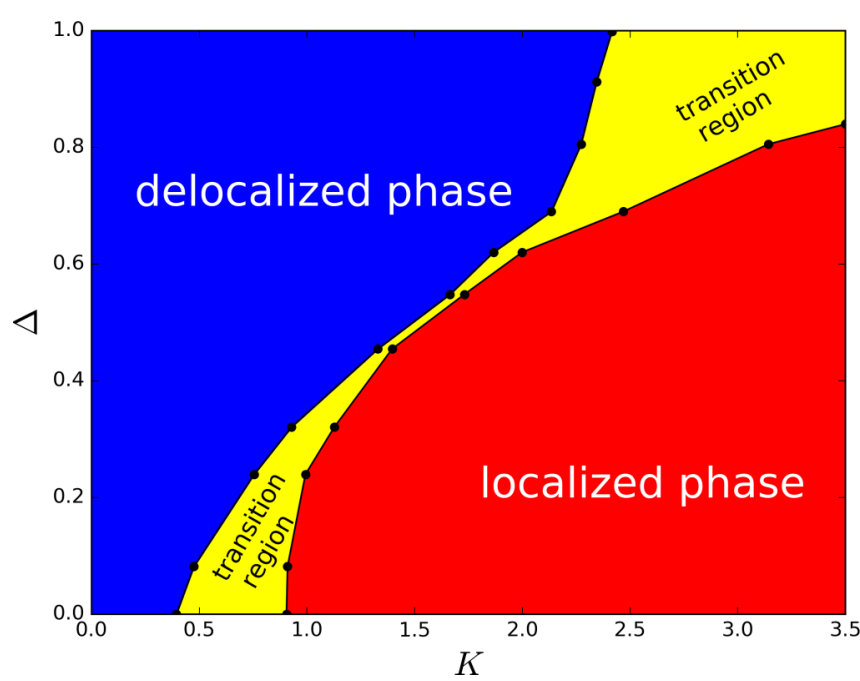

FIG. 4. Phase diagram of the Aubry transition. The variance of the central particle is displayed in $\Delta-K$ space. Red region: localized phase. Blue region: delocalized phase. Yellow region: intermediate phase. For the sake of definiteness, we have quantitatively defined phase boundaries as follows: $\sigma_{0}^{2}<0.015 a^{2}, 0.015 a^{2}<\sigma_{0}^{2}<$ $0.11 a^{2}$, and $\sigma_{0}^{2}>0.11 a^{2}$ for the delocalized, intermediate, and localized phases, respectively.

below this asymptotic value because the lack of localization of the other particles above the threshold $K_{c}$ broadens the central-particle wave function around the two peaks.

\section{The phase diagram}

In Fig. 4, we display in a single graph the system behavior as a function of the two relevant parameters, the potential strength $K$ and the degree of incommensurability $\Delta$. This plot, derived from the central-particle variance $\sigma_{0}^{2}$, can be considered a phase diagram that summarizes the general findings discussed so far. Larger incommensuration leads to larger values of the critical threshold $K_{c}$ above which the delocalized phase (blue region) is progressively lost, and the localized phase (red region) sets in. The size of the transition region (yellow region) progressively widens in the limit of large $K$ and $\Delta$ values, where incommensuration effectively fights the localizing effect imposed by the periodic optical potential.

A similar, although less pronounced, widening is observed for small incommensurations. We infer that this might be due to the tunneling processes, that are effective for $K \simeq K_{c}$ at all incommensuration values. If we had in Fig. 4 considered instead the Binder cumulant as the indicator for the transition, the width in the low $K-\Delta$ region would be greatly reduced, indicating that the variance tends to overestimate the quantum effects. In essence, both incommensuration and quantum effects act to increase the fluctuations and resist localization, as intuitively expected.

\section{Spectroscopic observables}

Up to this point, we have characterized the transition for the whole system via the behavior of the central particle. As already discussed, on physical grounds this is exactly what 


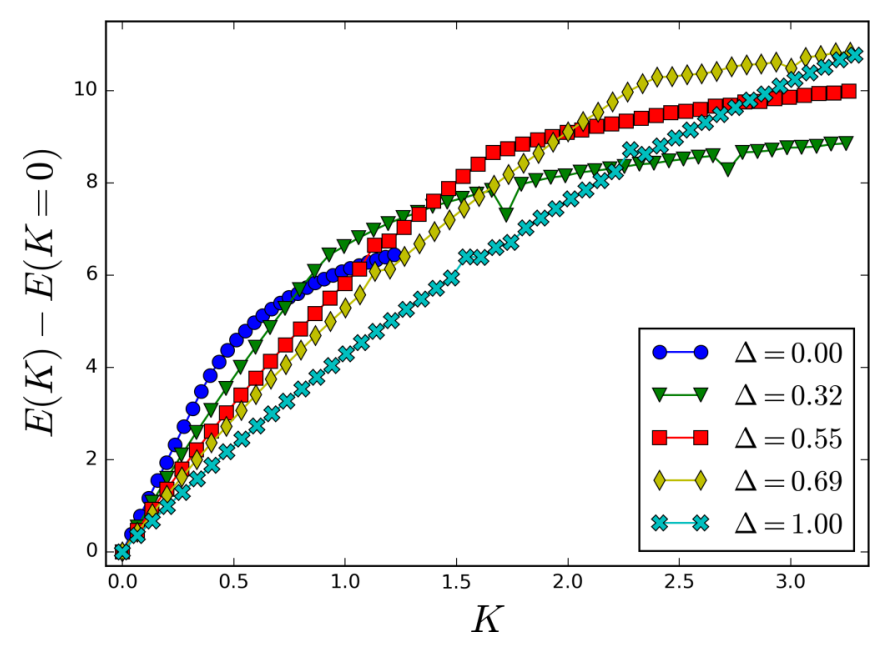

FIG. 5. Behavior of the system energy as a function of potential depth $K$ for different incommensuration parameter values $\Delta$, measured relative the energy for $K=0$. Once the transition occurs the energy growth-rate slows down, a sign that all the particles are localized in potential minima, so that the energy does not sensitively depend on $K$. Similarly to the variance in bottom Fig. 3, for the maximally incommensurate case the decrease in slope is never reached and the steepest behavior occurs for intermediate incommensurations.

happens in the fully commensurate case, where the springs can be ignored. However, we have already seen that with increasing incommensuration the transition becomes less sharp. In this and the next sections, we quantitatively trace the origin of this behavior to the collective properties of the system, and to the behavior of the other particles. We first consider the evolution of the total energy of the system, that, being a global quantity, should contain signatures of the combined behavior of all particles.

The transition is more evident if instead of displaying the total energy, we consider the difference in energy between the lattice potential being switched on $(K \neq 0)$ and off $(K=0)$. Experimentally, this can be measured directly by transferring the ions to a different internal state where they do not experience the light shift of the optical lattice. In this quantity also, a remarkable change occurs around the same critical $K_{c}$ as the one inferred from the maximum variance and the $\mathrm{BC}$ analysis. This is displayed in Fig. 5, where the change is seen as a "knee" in the curve, i.e., a change in the slope of the growth rate of differential energy with lattice potential $K$. This behavior can be understood with the following argument: when $K$ is increased in the sliding phase below $K_{c}$, the particles tend to be pushed closer to each other while trying to localize in the minima of the optical lattice. During this process, the energy must increase as a result of the interparticle elastic forces. No appreciable differences are encountered in this region between the quantum model and the calculation for the classical system (see Appendix B). On the other hand, after the transition has occurred, and the particles begin to tunnel between different minima, the elastic energy grows less quickly in the quantum model. We remark that the energy difference in Fig. 5 and the maximum variance in bottom Fig. 3 share similar behavior in the maximally incommensurate regime with $\Delta=1$, where no transition is observed, even after extending simulations to considerably larger values of $K$.

Finally, it is worthwhile to compare the energy difference of Fig. 5 with its analogous in the classical case. In the latter case, the energy curves exhibit $K$ dependencies which look qualitatively very similar to those of Fig. 5 (see Appendix C), with the difference that for large fixed $K$ the energy difference first increases with increasing incommensuration strength $\Delta$ until it reaches a maximum at $\Delta \sim 0.5$ and then decreases again while $\Delta$ approaches 1 . Furthermore, in the classical case, the second $K$ derivative of the energy, $\partial^{2} E / \partial K^{2}$, provides a very clear indicator of the transition (see Appendix C). Indeed, $\partial^{2} E / \partial K^{2}$ exhibits a sudden jump at the value of $K$ at which the central particle moves away from the origin. A similar analysis in the quantum case would be certainly very interesting, as it would provide a many-particle indicator of the transition which goes beyond the single- and two-particle quantities here analyzed, and it could be exploited even in the thermodynamic limit $N \rightarrow \infty$. However, within the present PIMC algorithm, it is not possible to extract a reliable numerical derivative of the energy differences in Fig. 5 due to the noise level present in the data.

\section{E. Hull function}

A second quantity that reflects the behavior of all particles is the hull function, that is often used to characterize the incommensurate and commensurate phases [20,33]. In our quantum system, this is the average position $\left\langle x_{0}\right\rangle$ of the central particle as a function of the phase offset $\Phi$ between the periodic potential and the harmonic trap potential, see Eq. (2). Here and in the following, the averaging refers to weighting with the quantum-mechanical probability density $\left|\Psi\left(x_{-(N-1) / 2}, \ldots, x_{(N-1) / 2}\right)\right|^{2}$, as determined from the $N$-ion wave function $\Psi$.

Below a threshold $\left(K<K_{c}\right)$, the particles are approximately uniformly arranged, and float on top of the lattice potential. The hull function in this region is continuous and monotonically increasing. In this characterization, the classical Aubry transition appears as a breaking of the analyticity of the hull function. Indeed we find that also for the finite quantum system, for $K>K_{c}$, as displayed in Fig. 6, gaps in the hull function open up at each value of translation $\Phi$ for which one of the particles would be located at a local maximum of the optical potential. The appearance of multiple gaps signals the occurrence of incommensurations, where the particles no longer slip synchronously. We notice, however, that here only two out of the three possible gaps open up, and that the tertiary gaps disappear due to lack of localization of the particles at the edges of the chain. This is evident from the bottom-right inset, displaying the BCs for the first three particles (labelled as $p_{2}, p_{1}, p_{0}$ starting from the edge of the chain), as a function of $K$ : at $K=3.34$ as in the main figure, particle 2 remains delocalized while particle 0 is already localized. This behavior can be contrasted with the classical case displayed for the very same set of parameters in the top-left inset of the figure, where all the three gaps are seen to open up. The corresponding behavior has also been observed experimentally (see Figs. 1(e) and 1(f) in Ref. [33]). Note that the number of discontinuities in the hull function corresponds 


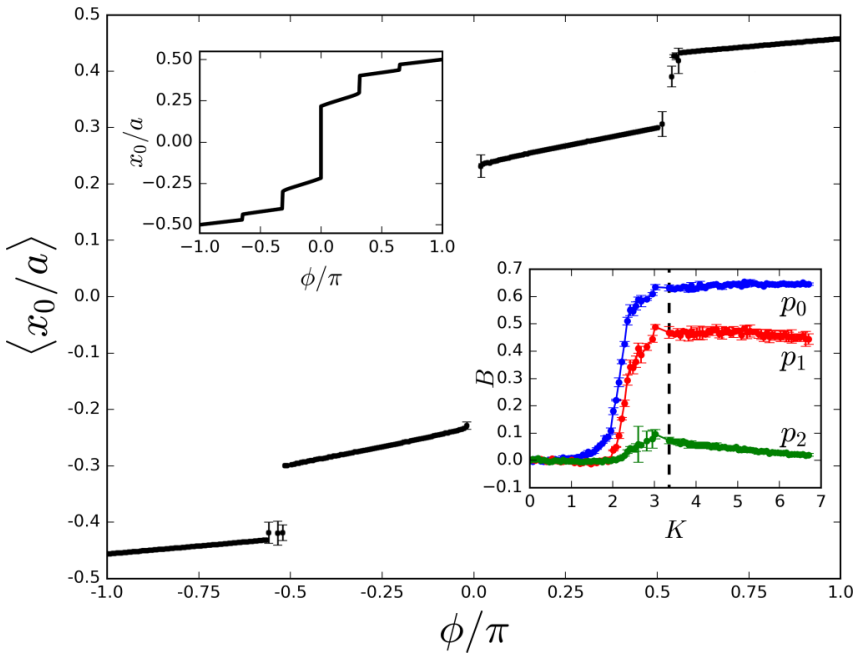

FIG. 6. Hull function, i.e., the central particle average position as a function of the phase shift $\Phi$ for the maximally incommensurate system and $K=3.34>K_{c}$ above the pinning transition according to the better suited Binder indicator. Gaps are seen to open at each value of $\Phi$ for which one of the particles has to overcome a maximum of the optical potential. For $K<K_{c}$, the hull function would be a continuous function. Top-left inset: the Hull function in the classical case for the same parameters set as in the main figure. Notice that here all the gaps open up, while in the quantum case the tertiary gaps disappear due to quantum tunneling. Bottom-right inset: Binder functions for the first three particles $p_{0,1,2}$ as a function of $K$ : at $K=3.34$ as in the main figure (dashed vertical line) particle 2 remains delocalized while particle 0 is localized.

to the number $N$ of particles in the system and that, classically, in the large- $N$ limit the hull function eventually becomes a Devil's staircase or Cantor function [15].

\section{F. Correlations}

We now explicitly look at the behavior of the other particles by analyzing suitable two-particle correlations, defined as

$$
g^{(2)}(i, j)=\frac{\left\langle\left(x_{i}-\bar{x}_{i}\right)\left(x_{j}-\bar{x}_{j}\right)\right\rangle}{\sqrt{\sigma_{i}^{2} \sigma_{j}^{2}}} .
$$

Figure 7 illustrates the behavior of $g^{(2)}(i, j)$ in the two extreme cases of a maximally commensurate (left panel) and of a maximally incommensurate (right panel) system. In the commensurate case, above a critical $K$ value all the correlations saturate to 1 , signaling a highly coherent behavior. We notice that, when compared to the commensurate case, all the pair correlations in the incommensurate regime are smoother and never saturate to 1 , a strong sign that while the central particle undergoes the pinning transition, the particles at the edges remain delocalized.

The correlation pattern between the particles is nontrivial. For example, correlations between the $(1,2)$ and the $(-1,1)$ pairs cross each other as a function of potential depth $K$. For small $K$, the $(1,2)$ correlation is larger because it involves two neighbors, while the $(-1,1)$ is a weaker second-neighbor correlation. However, for large $K$, the -1 and 1 particles undergo
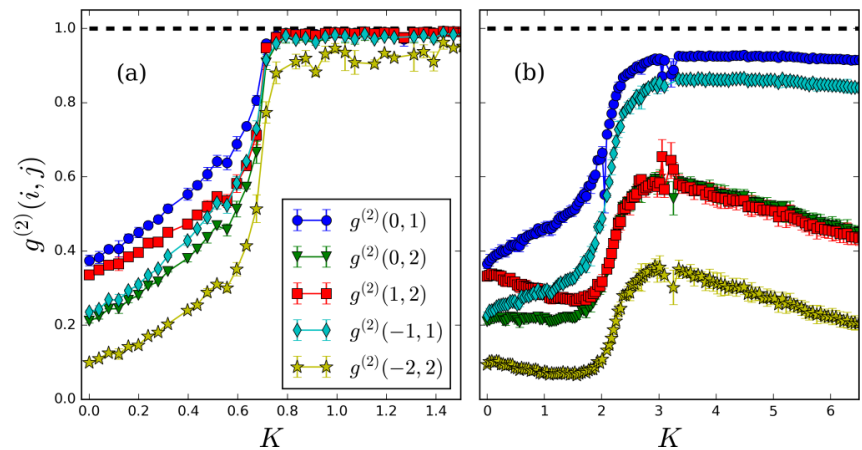

FIG. 7. Two-particle correlations. The particles are labeled such that 0 indicates the central particle, 1 and -1 its right and left first neighbors, etc. (a) (Almost) maximally commensurate case. (b) (Almost) maximally incommensurate case. The line $g^{(2)}(i, j)=1$ at which two particles are maximally correlated is displayed in both figures.

the pinning transition, and become highly correlated, while the outer particles $2,-2$ remain delocalized. We finally notice that, similarly to what happens to the $\mathrm{BCs}$ and the maximum variances, the correlations involving particles $1,-1,2$, and -2 decrease for large $K$ values.

\section{G. Temperature and size effects}

Finally, we verify to what extent the observed behavior across the transition is influenced by finite temperature or the finite size of the system. Regarding the role of temperature, we have compared simulations for two temperatures differing by one order of magnitude, with all other parameters kept fixed. As detailed in the SM (see Appendix B), no significant differences have been found in the three indicators of the transition, the BC, maximum-variance, and the system energy. We thus conclude that quantum effects can be observed even above the lowest accessible temperature.

Second, the harmonic confinement favors delocalization of the most external particles, and thus one might ask whether the effects found in the calculations would persist in a homogeneous system. In order to mimic the system in the absence of external confinement under stable conditions, we have performed the same simulations in a ring geometry with the harmonic confinement switched off. Figure 8 displays the Binder cumulants, variances, and correlation functions under maximally incommensurate conditions. After comparison of the three data sets to the corresponding observables illustrated in Figs. 3 and 7, we infer that the localization is not a result of finite-size conditions. In particular, the observed behavior of the covariances in the bottom panel of Fig. 8 strongly supports the genuine quantum origin of the correlation functions in Fig. 7, involving all the particles but the central one in a maximally incommensurate setup. Notice that the we have limited the numerical experiment to $K>1$ values, because the absence of harmonic confinement makes the practical simulation unstable for shallow external potential wells. In addition (see Appendix B), we observe the persistence of the energy behavior in Fig. 5. 

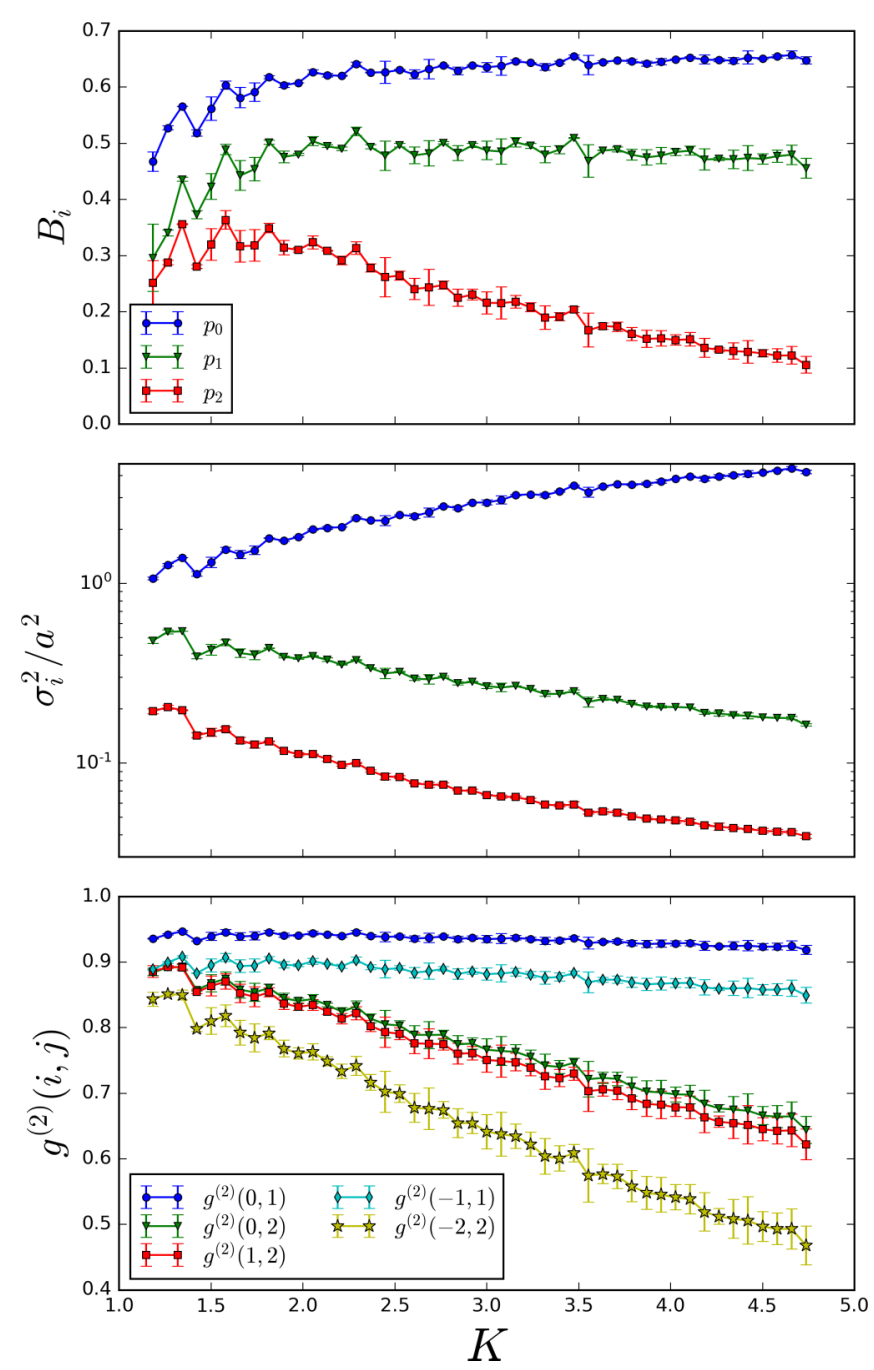

FIG. 8. Finite-size effects. Binder cumulants (upper left), variances (upper right), and correlations (bottom) for a system in a ring geometry, without harmonic confinement, i.e. mimicking an infinite system. The calculations have been performed for the maximally incommensurate case.

\section{PROSPECTS FOR THE EXPERIMENTAL OBSERVATION OF QUANTUM EFFECTS IN THE AUBRY TRANSITION}

The parameters used for the calculations described above are in the same range as in the experiment of Bylinskii et al. [35]. In particular, the temperatures where quantum effects are observable have already been reached in the experiment using Raman sideband cooling [42], and even lower temperatures can be reached using cooling on a narrow optical-clock transition [43]. Therefore finite temperature should not constitute an obstacle that would prevent the observation of a quantum Aubry transition.

Among the quantum signatures of the Aubry transition discussed in the current work, the energy of the system is the most directly accessible. It can, e.g., be directly measured spectroscopically by probing a transition to an internal state in which the ions do not experience the periodic optical potential. For instance, in the experiment by Bylinskii et al. [35], the periodic potential was generated by an optical lattice relatively near detuned to a transition ${ }^{2} S_{1 / 2} \rightarrow{ }^{2} P_{1 / 2}$. This optical lattice creates a periodic potential for the electronic ground state ${ }^{2} S_{1 / 2}$, but not for the long-lived electronic excited states (clock state [43]) ${ }^{2} D_{5 / 2}$. Thus with one laser beam addressing all ions, one could directly probe the energy difference between the system with optical potential (in the ground state ${ }^{2} S_{1 / 2}$ ) to the system without optical potential (in the clock state ${ }^{2} D_{3 / 2}$ ). This would enable one to map out a curve as shown in Fig. 5, and verify the transition spectroscopically. Alternatively, one can also probe excitations of the system by optically addressing only a single ion and transferring it to the state ${ }^{2} D_{3 / 2}$ where it does not experience the periodic potential.

The optical resolution with which the atom position can be directly measured, on the other hand, is typically insufficient to determine the $\mathrm{BC}$ or the change of the variance of the atomic position across the Aubry transition. However, as demonstrated in the experiments [33-35], the atomic fluorescence during the continuously applied laser cooling depends sensitively on the position of the atom relative to the optical lattice. Using this tool, it has been possible to determine the average positions of all the ions across the transition, with resolution well below the lattice period $a$ [35]. Extending this technique, it may be possible to use the statistics of the continuously detected atomic fluorescence light to infer the position distribution for each atom, and to reconstruct the $\mathrm{BC}$ and the variances of the atomic positions.

\section{CONCLUSIONS}

In conclusion, we have performed PIMC simulations precisely reproducing the conditions of the experiment on an Aubry-type transition in a finite ion chain [33]. We have found that quantum effects in the Aubry transition emerge in two different observables never considered before as indicators, namely, the Binder cumulant, and a suitably devised spectroscopic technique measuring the difference in energy between the system with and without exposure to the optical lattice. The maximum variance may be added as a third signature of the transition. For all three observables, a steep, sudden change of behavior consistently occurs at a given critical value of the periodic potential $V_{c}$ depending on the incommensuration parameter $\Delta$ as summarized in Fig. 4. At least some of these quantities can be measured in the experiment [35], leading to a promising route to identifying the impact of quantum effects on the Aubry transition. Even more remarkably, the transition appears to be robust against finite-size effects, and may be discernible even in relatively small chains of ions. It would be interesting to follow the evolution of the transition with increasing ion number, although the experiment would eventually be limited by stability conditions, while our particular PIMC is limited by increasing simulation times. References [20,36] comment that the transition appears to be second order. However, we feel that a definite answer to this question can only be given after identifying a suitable order parameter, as none of those considered in literature is fully suitable. In this respect, a useful tool can be the study of structure factors, which, in contrast to the single- or two-particle quantities analyzed in this paper, contain information about the global behavior of the system. Another suitable quantity 
for this purpose is the quantum fidelity matrix [44-46], that in our case is essentially represented by the second derivative of the overlap between two ground states at two slightly different values of $V$ with respect to the optical potential height $V$. This parameter could be sampled during the whole PIMC simulation. The quantum fidelity matrix could be particularly useful to study the properties of the phase transition, also while considering different system sizes, since it does not require identification of an order parameter. In particular, a transition would be unequivocally signaled by a peak in the behavior of the quantum fidelity matrix as a function of $V$.

The focus of the present work is on the observability of quantum effects, a nontrivial task in the presence of Coulomb long-range interactions [47,48], and to augment existing work on the quantum Aubry transition [20,36]. It paves the way for studies along a number of different directions to be explored in future combined theoretical-experimental effort [48]. Those include the possibility of elucidating the universality class of the transition while tuning the degree of quantumness, or to assess whether and to which extent the transition can be related to the paradigm of many-body localization. Finally, the very general nature of the Aubry transition, as occurring in so many diverse classical and quantum systems, can in principle be probed for different microscopic mechanisms. This would be a natural operation to be performed, e.g., using available platforms of ultracold atomic quantum gases. In fact, we may envisage a number of different microscopic mechanisms. For example, using ions in optical cavities, one may build the system of Ref. [29] and investigate the robustness of mechanical stability against quantum dynamics, or else engineer the quantum reservoir. The cases of dipolar and contactlike interactions can be especially interesting in shining light on whether and how the interaction range affects the transition, with Rydberg-atom systems covering the intermediate interaction range. Finally, by controlling Rydberg atoms inside optical cavities, one can access an intriguing scenario where the infinite-range nature of the effective interatomic interaction mediated by cavity photons is combined with the strong and coherent medium-range interaction between Rydberg atoms.

\section{ACKNOWLEDGMENTS}

This work has been supported in part by the NSF (Grant No. PHY-1806765) and NSF CUA (Grant No. PHY-1734011), and by the MITUniPi Program. We thank Giovanna Morigi and Massimo D'Elia for very fruitful discussions. M.L.C. and P.M.B. are grateful to MIT for hospitality.

\section{APPENDIX A: METHODS}

In this section, we provide additional details on the simulation method. In a path-integral Monte Carlo (PIMC), a Markov chain Monte Carlo (MCMC) [41] is used in order to generate the path integrals of the physical system. In this formulation, the partition function of the system is rotated in the Euclidean space performing a Wick rotation $t \rightarrow i \tau$ in the classical action. Thus time evolution occurs in an imaginary time axis with periodic boundary conditions and length given by $\hbar / k_{B} T$, where $T$ is the temperature of the system. In this manner, a configuration of the system is represented by a collection of several (closed-loop) paths, each one describing the time evolution of a particle in the physical space. Configurations are generated numerically through a MCMC, and are sampled according to the probability distribution of the system which depends on the action. This allows one to compute the observables of interest of the system from the sample.

To this aim, we first write the classical action in dimensionless units. In our case, the discretization adopted is essentially a generalization of that used by Creutz and Freedman in their seminal paper in Ref. [49]. The imaginary time direction is discretized introducing a time step of length $\Delta \tau=\hbar \beta / N_{\tau}$, which splits the total length $\hbar / k_{B} T=\hbar \beta$ in $N_{\tau}$ time slices, and a left-derivative operator is adopted. The action of the system reads as

$$
\begin{aligned}
\frac{S_{\mathrm{discr}}}{\hbar}=\Delta & \tau \sum_{\mu=0}^{N_{\tau}-1}\left\{\sum _ { i = - ( N - 1 ) / 2 } ^ { ( N - 1 ) / 2 } \left[\frac{1}{2}\left(\frac{X_{i}(\mu+1)-X_{i}(\mu)}{\Delta \tau}\right)^{2}\right.\right. \\
& \left.+\frac{1}{2} \tilde{\omega}_{0}^{2}\left(X_{i}(\mu)-\Phi\right)^{2}+V\left[1+\cos \left(X_{i}(\mu)\right)\right]\right] \\
& \left.+\frac{1}{2} \sum_{i \neq j} \frac{Q^{2}}{\left|X_{i}(\mu)-X_{j}(\mu)\right|}\right\},
\end{aligned}
$$

where all the quantities are expressed in dimensionless units. In the present equation, latin indices enumerate the particles while greek ones the refer to the (imaginary) time steps, with the boundary condition $X_{i}\left(N_{\tau}\right)=X_{i}(0) \forall i$.

Configurations of the systems are sampled starting from an initial ordered state and then proposing a random spatial move for each particle at each time step, sweeping the imaginary trajectory of each particle one after the other. The MCMC chain of system states is constructed according to the METROPOLIS algorithm [41,50]: the proposed move is accepted or rejected with probability proportional to the ratio of the statistical weights of the new and the old configuration. Further details about the algorithm itself and the discretization procedure can be found in Ref. [49]; we refer to the latter for details and for a discussion of the underlying physics. In our case, we apply the PIMC to a system of interacting ions in a one-dimensional space. In principle, because of the fermionic nature of the particle of the system, anticommutation rules must be taken into account. For example, a sign swap is needed in the PI formulation when treating configurations with crossing particle paths. However, in our case the situation is easier and less involved because, as shown by Girardeau [51], in a one-dimensional space, fermions are equivalent to hard-core bosons, and the strong repulsive Coulomb interaction keeps the particles away from each other so that the individual wave functions never overlap. Then it suffices to simply reject states in which pairs of particle trajectories are crossing each other.

Observables of interest for the system are suitably defined and measured directly on the sample of configurations obtained from the MC chain. However, it is important to notice that both the statistical analysis of the data and the extraction of the values of the observable is not straightforward and must be handled with care. First of all, data generated using MCMC contain autocorrelations [41], which must be took 
into account in order to obtain reliable and unbiased estimates. In order to do so, the extraction of the observable is performed each $n$ swipes of all particle imaginary-time trajectories, with $n$ suitably chosen so as to decrease the correlation between two subsequent measures. Moreover, a blocking technique has been used, in order to take into account-and to further reduce-correlations in the data samples. In addition, a jackknife resampling method has been used to obtain a reliable estimation of the variance associated to the values of the observables [52-54].

A second and very relevant aspect is related to the extent to which the adopted system discretization affects the measurements. In order to obtain true physical estimates of the values of the observables, a continuum limit is performed: both the simulations and the analysis are carried out several times for different decreasing values of the time step $\tau$ at the same physical temperature, and then the continuum value $\tau \rightarrow 0$ of the observable is extrapolated through a fit procedure. In addition, in order to efficiently deal with the tunneling processes arising when the minimum of the harmonic trap coincides with a maximum of the optical potential, we have devised the algorithm in a way that after $m$ ordinary METROPOLIS moves (with $m$ suitably chosen), a new type of move is proposed. Indeed, with the aim of a correctly sampling all the configuration space, we have modified the algorithm so that it proposes as a new configuration the reflection of the actual configuration with respect to the minimum of the harmonic trap. It has been seen and checked that this kind of update indeed reduces autocorrelation effects in the data.

\section{APPENDIX B: FINITE-SIZE EFFECTS AND THE ROLE OF FINITE TEMPERATURE}

In this section, we report on the numerical checks that we have performed, in order to retain control of the effects due to finite size and finite temperature, and verify that the conclusions of the manuscript remain unaffected.

The effects due to finite size can ideally be checked by removing the harmonic confinement. Due to the presence of

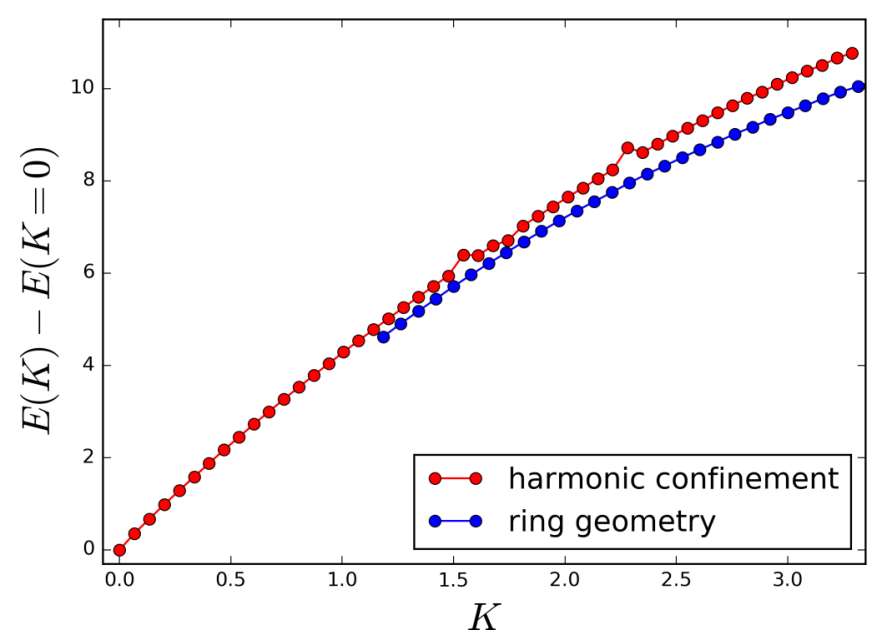

FIG. 9. Finite-size effects. Behavior of the energy in a maximally incommensurate system for two different geometries: the harmonic confined linear system and a system on a ring.
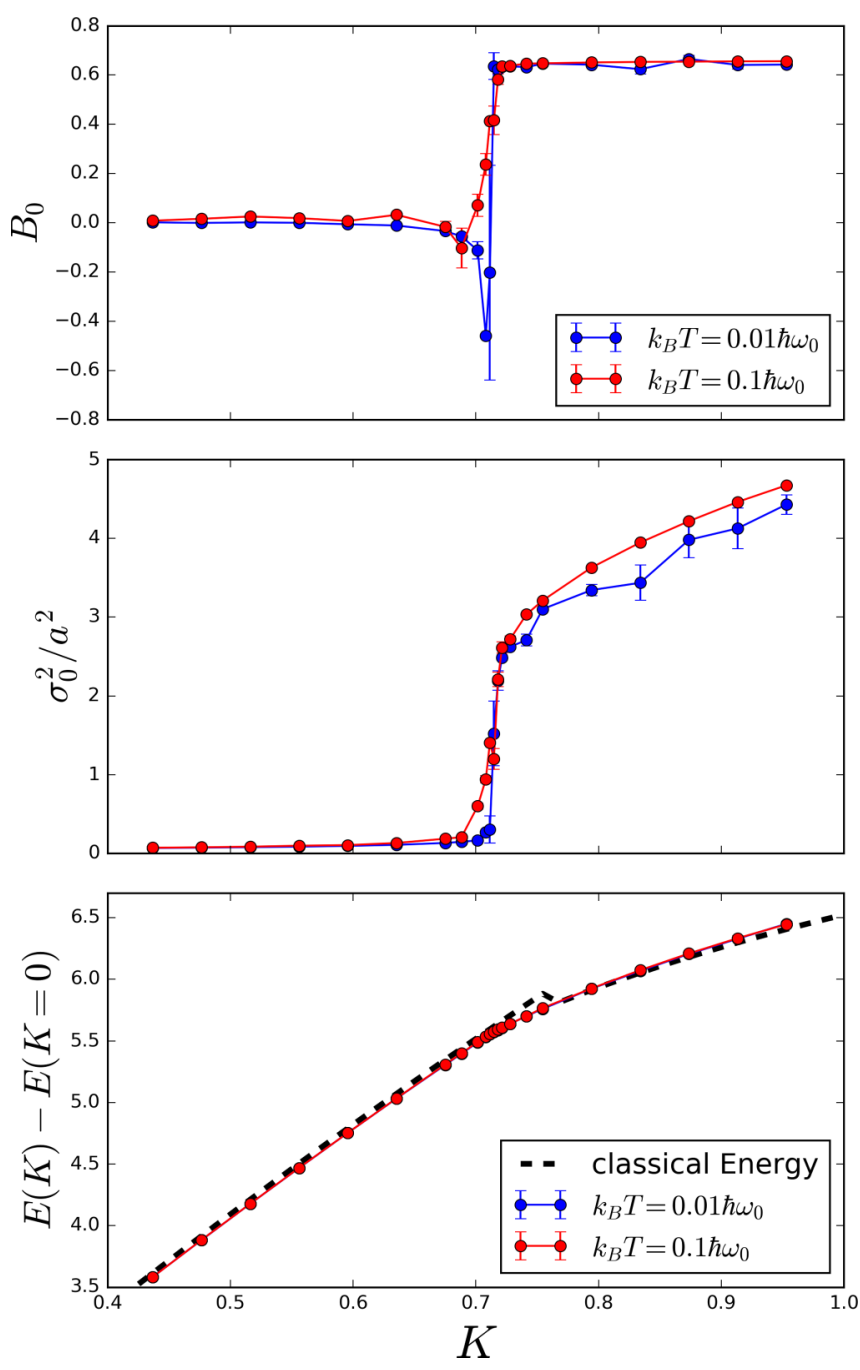

FIG. 10. Effect of temperature. Central particle Binder cumulant (top), variance (middle), and energy (bottom) for two different temperatures as specified in the legend.

the Coulomb repulsion, this is best performed by placing the ions in a ring geometry mimicking an infinite system. In Fig. 9, we confirm that the transition to the incommensurate phase cannot be inferred from the behavior of energy, due to the fact that the most external particles never localize.

We now provide evidence that the physics discussed in the main text is not masked nor significantly affected by temperature, within the range typical of the experiment [33]. We report in Fig. 10 the Binder cumulants, variances, and energy for two values of temperature that differ by one order of magnitude, i.e., $k_{B} T=0.01 \hbar \omega_{0}$ and $0.1 \hbar \omega_{0}$. From the behavior of the three observables, it is evident that at these low temperatures typical of the experiment, smoothing of the transition due to thermal fluctuations is not significant. In addition, the energy plot in the bottom panel clarifies the role of quantum effects. Indeed, while below the transition the quantum and classical energies differ only by a constant, for large $K$ instead their behavior slightly differentiates, due to quantum tunneling.

We close by commenting on the strength of the correlations in coherently driving the behavior of all the particles, as it becomes apparent from Fig. 11, where simulated samples 


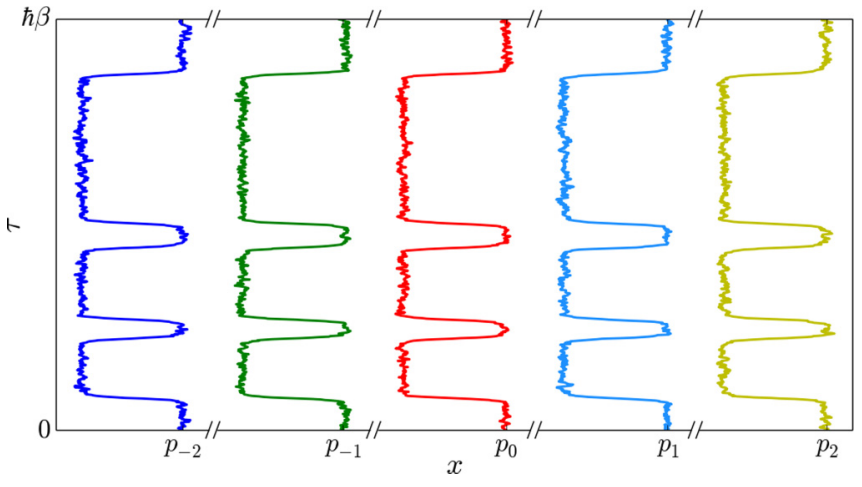

FIG. 11. Particle correlations. Sampled configuration of the imaginary time paths of the five particles (expressed in different colors), in the maximally commensurate case with $K>K_{c}$. The strong correlations among the particles drive coherent behavior.

of the imaginary-time paths of the five ions are represented under maximally commensurate conditions. From the figure it is evident how the tunneling processes of all the particles are sharply locked, while imaginary-time flows. This behavior witnesses the occurrence of instanton configurations, as also analyzed in Ref. [21]. Notice that such a striking correlated behavior along imaginary time, can be mapped into a lowerdimensional (1D) random-spin Ising model with transverse field via the Suzuki-Trotter method $[55,56]$.

\section{APPENDIX C: ENERGY IN THE CLASSICAL CASE}

In this section, we show a computation of the difference in energy between the system with and without exposure to the optical lattice in the classical system at zero temperature. In this case, we define the system energy as the minimum of the potential energy

$$
E_{c l}(K) \equiv \min _{\left\{x_{i}\right\}} V_{\text {pot }}\left(\left\{x_{i}\right\} ; K\right),
$$

with the potential energy $V_{\text {pot }}$ defined as

$$
\begin{aligned}
V_{\mathrm{pot}}\left(\left\{x_{i}\right\} ; K\right)= & \sum_{i=-(N-1) / 2}^{(N-1) / 2}\left\{\frac{1}{2} \frac{m \omega_{0}^{2}}{\gamma} x_{i}^{2}+K\left[1+\cos \left(\frac{2 \pi}{a} x_{i}\right)\right]\right\} \\
& +\sum_{i, j>i} \frac{e^{2}}{\gamma} \frac{1}{\left|x_{i}-x_{j}\right|},
\end{aligned}
$$

with $\gamma$ defined in the main text.

In Fig. 12, we show the quantity $E_{c l}(K)-E_{c l}(K=0)$ for different choices of the incommensuration parameter $\Delta$

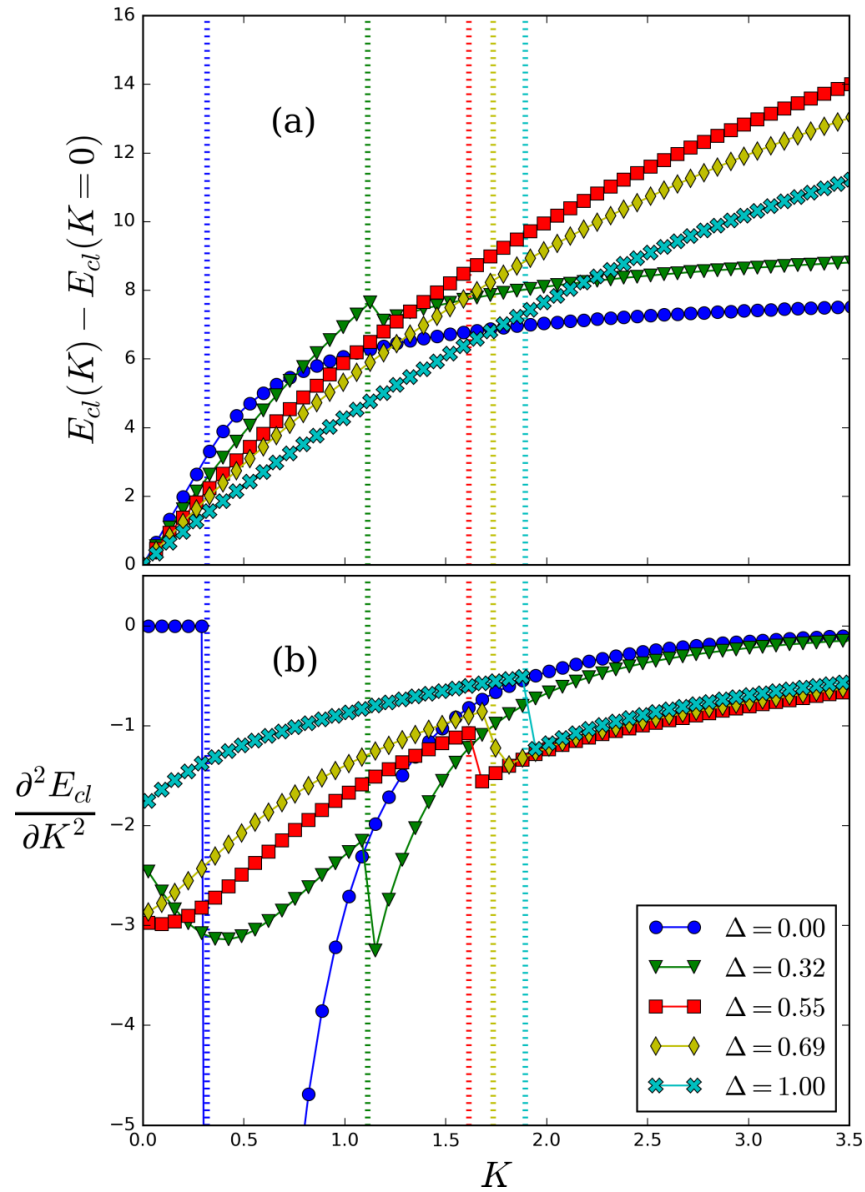

FIG. 12. (a) Classical energy for different choices of the incommensuration parameter $\Delta$, as in the legend. (b) Second derivative with respect to $K$ of the classical energy as a function for various values of $\Delta$. We notice that the second derivative exhibits a jump at the critical optical potential height $K_{c}$, at which the Aubry transition occurs. The values of $K_{c}$ for each value of $\Delta$ are reported in both panels as dotted lines.

(which can be varied by fine-tuning $\omega_{0}$ ), as well as its second derivative with respect to $K$. In the energy, we notice no qualitatively different behavior compared to the quantum case shown in the main text, except for the fact that for large $K$ the energy seems to increase by increasing $\Delta$ until it reaches a maximum at $\Delta \sim 0.5$ and then decreases again, while in the quantum case we see a monotonic behavior in $\Delta$. More interesting is the second derivative of the energy, which shows a sudden jump when the transition occurs. The size of the jump seem to decrease with increasing incommensuration strength up to $\Delta \sim 0.5$, when it starts increasing again.
[1] S. Sachdev, Quantum Phase Transitions (Cambridge University Press, Cambridge, 2000).

[2] G. Grüner, The dynamics of charge-density waves, Rev. Mod. Phys. 60, 1129 (1988).

[3] Y. Mo, K. Turner, and I. Szlufarska, Friction laws at the nanoscale, Nature 457, 1116 (2009).
[4] O. M. Braun and Y. S. Kivshar, The Frenkel-Kontorova Model: Concepts, Methods, and Applications (Springer, Berlin, Heidelberg, 2004).

[5] O. Braun and A. Naumovets, Nanotribology: Microscopic mechanisms of friction, Surf. Sci. Rep. 60, 79 (2006). 
[6] P. M. Chaikin and T. C. Lubensky, Principles of CondensedMatter Physics (Cambridge University Press, Cambridge, 2000).

[7] V. Bormuth, V. Varga, J. Howard, and Schäffer, Protein friction limits diffusive and directed movements of kinesin motors on microtubules, Science 325, 870 (2009).

[8] C. K. Chiang, C. R. Fincher, Y. W. Park, A. J. Heeger, H. Shirakawa, E. J. Louis, S. C. Gau, and A. G. MacDiarmid, Electrical Conductivity in Doped Polyacetylene, Phys. Rev. Lett. 39, 1098 (1977).

[9] P. Bak, Commensurate phases, incommensurate phases and the devil's staircase, Rep. Prog. Phys. 45, 587 (1982).

[10] K. Kürten and C. Krattenthaler, Multistability and Multi $2 \pi$ kinks in the Frenkel-Kontorova model: An application to arrays of Josephson junctions, Int. J. Modern Phys. B 21, 2324 (2007).

[11] E. Haller, R. Hart, M. J. Mark, J. Danzl, L. Reichsöllner, M. Gustavsson, M. Dalmonte, G. Pupillo, and H. C. Nägerl, Pinning quantum phase transition for a Luttinger liquid of strongly interacting bosons, Nature (London) 466, 597 (2010).

[12] M. Atala, M. Aidelsburger, M. Lohse, J. T. Barreiro, B. Paredes, and I. Bloch, Observation of chiral currents with ultracold atoms in bosonic ladders, Nature Phys. 10, 588 (2014).

[13] E. Orignac, R. Citro, M. D. Dio, S. D. Palo, and M.-L. Chiofalo, Incommensurate phases of a bosonic two-leg ladder under a flux, New J. Phys. 18, 055017 (2016).

[14] L. Consoli, H. J. F. Knops, and A. Fasolino, Onset of Sliding Friction in Incommensurate Systems, Phys. Rev. Lett. 85, 302 (2000).

[15] S. Aubry, The twist map, the extended Frenkel-Kontorova model and the devil's staircase, Phys. D 7, 240 (1983).

[16] T. A. Kontorova and Y. I. Frenkel, On the theory of plastic deformation and twinning. II, Zh. Eksp. Teor. Fiz. 8, 1340 (1938).

[17] F. C. Frank and J. H. Van der Merwe, One-dimensional dislocations. I. Static theory, Proc. R. Soc. London A 198, 205 (1949).

[18] S. R. Sharma, B. Bergersen, and B. Joos, Aubry transition in a finite modulated chain, Phys. Rev. B 29, 6335 (1984).

[19] F. Borgonovi, I. Guarneri, and D. Shepelyansky, Destruction of classical cantori in the quantum Frenkel-Kontorova model, Z. Phys. B. 79, 133 (1990).

[20] B. Hu and B. Li, Quantum Frenkel-Kontorova model, Phys. A 288, 81 (2000).

[21] O. V. Zhirov, G. Casati, and D. L. Shepelyansky, Quantum phase transition in the Frenkel-Kontorova chain: From pinned instanton glass to sliding phonon gas, Phys. Rev. E 67, 056209 (2003).

[22] Y. Ma, J. Wang, X. Xu, Q. Wei, and S. Kais, Quantum phase transition in one-dimensional commensurate FrenkelKontorova model, J. Phys. Soc. Jpn. 83, 124603 (2014).

[23] F. R. Krajewski and M. H. Muser, Quantum dynamics in the highly discrete, commensurate Frenkel Kontorova model: A path-integral molecular dynamics study, J. Chem. Phys. 122, 124711 (2005).

[24] B. Hu and J. X. Wang, Density-matrix renormalization group study of the incommensurate quantum Frenkel-Kontorova model, Phys. Rev. B 73, 184305 (2006).

[25] V. Pokrovsky and A. Virosztek, Solitary wave solutions of nonlocal sine-Gordon equations, J. Phys. C 16, 4513 (1983).
[26] O. M. Braun, Y. S. Kivshar, and I. I. Zelenskaya, Kinks in the Frenkel-Kontorova model with long-range interparticle interactions, Phys. Rev. B 41, 7118 (1990).

[27] H. Landa, C. Cormick, and G. Morigi, Kinks in the FrenkelKontorova model with long-range interparticle interactions, Condens. Matter 5, 35 (2020).

[28] P. Silvi, G. De Chiara, T. Calarco, G. Morigi, and S. Montangero, Full characterization of the quantum linear-zigzag transition in atomic chains, Ann. Phys. 525, 827 (2013).

[29] C. Cormick and G. Morigi, Structural Transitions of Ion Strings in Quantum Potentials, Phys. Rev. Lett. 109, 053003 (2012).

[30] D. A. Gangloff, A. Bylinskii, and V. Vuletić, Kinks and nanofriction: Structural phases in few-atom chains, Phys. Rev. Research 2, 013380 (2020).

[31] T. Fogarty, C. Cormick, H. Landa, V. M. Stojanović, E. Demler, and G. Morigi, Nanofriction in Cavity Quantum Electrodynamics, Phys. Rev. Lett. 115, 233602 (2015).

[32] P. Bak and R. Bruinsma, One-Dimensional Ising Model and the Complete Devil's Staircase, Phys. Rev. Lett. 49, 249 (1982).

[33] A. Bylinskii, D. Gangloff, I. Counts, and V. Vuletić, Observation of Aubry-type transition in finite atom chains via friction, Nat. Mater. 15, 717 (2016).

[34] D. Gangloff, A. Bylinskii, I. Counts, W. Jhe, and V. Vuletić, Velocity tuning of friction with two trapped atoms, Nat. Phys. 11, 915 (2015).

[35] A. Bylinskii, D. Gangloff, and V. Vuletić, Tuning friction atom-by-atom in an ion-crystal simulator, Science 348, 1115 (2015).

[36] A. Benassi, A. Vanossi, and E. Tosatti, Nanofriction in cold ion traps, Nat. Commun. 2, 236 (2011).

[37] D. Mandelli, A. Vanossi, and E. Tosatti, Stick-slip nanofriction in trapped cold ion chains, Phys. Rev. B 87, 195418 (2013).

[38] T. Pruttivarasin, M. Ramm, I. Talukdar, A. Kreuter, and H. Häffner, Trapped ions in optical lattices for probing oscillator chain models, New J. Phys. 13, 075012 (2011).

[39] I. García-Mata, O. V. Zhirov, and D. L. Shepelyansky, FrenkelKontorova model with cold trapped ions, Eur. Phys. J. D 41, 325 (2007).

[40] J. Kiethe, R. Nigmatullin, D. Kalincev, T. Schmirander, and T. E. Mehlstaubler, Probing nanofriction and Aubry-type signatures in a finite self-organized system, Nat. Commun. 8, 15364 (2017).

[41] H. J. Rothe, Lattice Gauge Theories: An Introduction, Lecture Notes in Physics (World Scientific, Singapore, 2012), Vol. 82.

[42] L. Karpa, A. Bylinskii, D. Gangloff, M. Cetina, and V. Vuletić, Suppression of Ion Transport due to Long-Lived Subwavelength Localization by an Optical Lattice, Phys. Rev. Lett. 111, 163002 (2013).

[43] P. Taylor, M. Roberts, S. V. Gateva-Kostova, R. B. M. Clarke, G. P. Barwood, W. R. C. Rowley, and P. Gill, Investigation of the ${ }^{2} S_{1 / 2}-{ }^{2} D_{5 / 2}$ clock transition in a single ytterbium ion, Phys. Rev. A 56, 2699 (1997).

[44] P. Zanardi and N. Paunković, Ground state overlap and quantum phase transitions, Phys. Rev. E 74, 031123 (2006).

[45] P. Zanardi, P. Giorda, and M. Cozzini, Information-Theoretic Differential Geometry of Quantum Phase Transitions, Phys. Rev. Lett. 99, 100603 (2007).

[46] L. Wang, Y.-H. Liu, J. Imriška, P. N. Ma, and M. Troyer, Fidelity Susceptibility Made Simple: A Unified Quantum Monte Carlo Approach, Phys. Rev. X 5, 031007 (2015). 
[47] A. Retzker, R. C. Thompson, D. M. Segal, and M. B. Plenio, Double Well Potentials and Quantum Phase Transitions in Ion Traps, Phys. Rev. Lett. 101, 260504 (2008).

[48] M. L. Chiofalo, G. Morigi, and V. Vuletić, ongoing work under the MIT-UNIPI program (2020).

[49] M. Creutz and B. Freedman, A statistical approach to quantum mechanics, Ann. Phys. 132, 427 (1981).

[50] W. K. Hastings, Monte Carlo sampling methods using Markov chains and their applications, Biometrika 57, 97 (1970).

[51] M. Girardeau, Relationship between systems of impenetrable bosons and fermions in one dimension, J. Math. Phys. 1, 516 (1960).

[52] B. Efron and C. Stein, The jackknife estimate of variance, Ann. Stat. 9, 586 (1981).
[53] M. H. Quenouille, Notes on bias estimation, Biometrika 43, 353 (1956).

[54] B. Efron, The Jackknife, the Bootstrap and Other Resampling Plans (Society for Industrial and Applied Mathematics, Philadelphia, 1982).

[55] M. Suzuki, Relationship between d-dimensional quantal spin systems and $(\mathrm{d}+1)$-dimensional ising systems: Equivalence, critical exponents and systematic approximants of the partition function and spin correlations, Prog. Theor. Phys. 561454 (1976).

[56] A. Dutta, G. Aeppli, B. K. Chakrabarti, U. Divakaran, T. Rosenbaum, and D. Sen, Quantum Phase Transitions in Transverse Field Spin Models: From Statistical Physics to Quantum Information (Cambridge University Press, Cambridge, 2015). 